\title{
The Internet: Challenges and Opportunities Faced by Modelers of Social Influence
}

\author{
Billy Campoli (william.campoli@ mail.mcgill.ca)
}

$5 / 05 / 2019$

\begin{abstract}
Even before the emergence of worldwide phenomena such as Brexit and the rise of populist right-wing politics, scholars have been long interested in the phenomena of social influence. In the last decade, the rise of online social media has posed new challenges and opportunities for researchers and modelers wishing to study social influence. In this review article, we trace back the study of social influence to its roots in early diffusionist theory. Then, in order to contextualize the issues faced by modern researchers of social influence, we attempt summarize it's more classic models and explanations. Finally, we shift our focus to the role of the internet, both as a source as a source of new opportunities and of new challenges for researchers interested in studying social influence.
\end{abstract}

Long before the invention of the internet, researchers have been interested in the study of social influence. Late 19th century scholars known as "diffusionists" attempted to study how information and ideas flowed and transmitted through people in society. It was through their early groundwork that modern models of social influence emerged. Today, much of the theories we have on social influence come from Agent Based Models (ABMs). Agent based models allow researchers to simulate or observe some global phenomena (such as conformity or polarization) from some base set of rules that are enforced upon the agents. Although highly simplified, some of these models can give researchers great insights into the underlying dynamics at play. The emergence of the internet introduces new dynamics in the study of social influence. In this paper we attempt to elucidate the ways in which the internet changes the dynamics of traditional models of social influence, and attempt to provide some tips and hints for modelers wishing to improve the ecological validity of their models online.

In the first part of the paper, we provide context for the study of social influence by tracing back it's study to diffusionist theory. We then give a broad overview of modern ABMs used to explain social influence. We conclude with a discussion of what ingredients these models are missing to fully accommodate for the "internet age" and discuss ways in which modelers could add additional ingredients to better accommodate them for this domain.

\section{Roots of Social Influence: The Diffusionists}

The study of social influence is a broad tradition, which touches on many fields including, but not limited to, sociology, psychology, computer science, data science, and anthropology. The roots of this tradition can be traced back to the early diffusionists of the late 19th century. Gabriel Tarde, one of the founding fathers of early diffusionist theory, was one of the first to systematically study the subject (Kinnunen 1996). In one of his landmark works les lois de l'imitation, Tarde posited imitation as the driving force in explaining human activity. Certain humans would develop innovations, or novel inventions, and these innovations would spread amongst society through the process of imitation (Kinnunen 1996). Although Tarde never explicitly used the term diffusion, he is oft considered one of the founders of the field (Thomas W. Valente 1995). Although some anthropologists and sociologists of the 1920s and 30s studied the diffusion of various innovations (such as postage stamps and 
of some consumer innovations), Thomas and Valente (1995) classify this as pre-paridgmatic work on the study of diffusion: that is, these early researchers were mainly conducting evaluation studies "that lacked a theoretical framework" (Thomas W. Valente 1995).

It wasn't until the 1940s that researchers began to develop formal theoretical models on the diffusion of innovations (Thomas W. Valente 1995). Thomas and Valente (1995) credit Ryan and Gross' (1943) study on the The diffusion of hybrid seed corn in two Iowa communities as the first to advance theoretical explanations on the process of diffusion, in contrast to the more evaluative studies of the past. Ryan and Gross (1943) laid the groundwork for the new paradigm through their investigations of: 1) the individual decision making process leading to adoption, 2) the role of information channels and sources, 3) the s-shaped adoption curve, and 4) the socioeconomic traits of the adopters (Thomas W. Valente 1995). Throughout the 1950s, diffusion research was confined mostly to rural sociologists (Thomas W. Valente 1995). In the 1960s, in part due to Rogers (1963) seminal work, Diffusion of innovations, the study of diffusion began to spread to other scientific disciplines. Rogers generalized the theory of diffusion past the domain of farming which paved the road for the study of diffusion in other domains (Rogers 1963; Thomas W. Valente 1995).

In the 1990s, agent-based models became one of the main techniques used to study social influence. Agent based models provided the benefits of being able to describe an emergent phenomena from the constituent agent interactions, giving a rich "bottom-up" explanation for how the phenomena occurs (Bonabeau 2002). Famous models such as Axelrod's (1997) The Dissemination of Culture: A Model with Local Convergence and Global Polarization showed that from a simple set of rules, local opinion clusters could form within a population, preventing it from reaching a consensus. Since Axelrod's famous publication, hundreds more ABMs have been developed in an attempt to explain social influence.

\section{Agent-Based Models of Social Influence}

In their literature review on models of social influence, Flache et al. (2017) classify the kinds of models seen in the literature into three broad classes. Models were grouped by the researchers on the basis of their formal implementation, rather than their theoretical underpinnings. That is to say, models of the same class need not share the same theoretical justifications (Flache et al. 2017).

The first class of models they identify are models of assimilative social influence. In models of assimilative social influence, global convergence on some idea or opinion is inevitably achieved despite the initially different belief states of the agents. When agents interact in these sorts of models, they will work to reduce opinion differences (Flache et al. 2017). Models of of assimilative social influence tend to use both sociological and psychological justifications for the rules that the agents are following. Sociological theories of conformity to group norms (Maccoby 1962) and psychological theories of cognitive dissonance reduction (Festinger 1957) are often used to justify these sorts of models. Models in this class can represent opinions as continuous (ie: as a real valued number representing the strength of the belief) or nominally (either the agent has or does not have the opinon). Formal implementations of models of assimilative social influence that use continous opinion representation tend to use averaging as a mechanism for opinion difference reduction. In these sorts of models, when an agent exerts influence over another agent, opinion difference is reduced by a mechanism of shifting the influenced agent's opinion toward the average of both agents' opinions. The amount an agent's opinion changes usually depends some connection weights between the agents which represents the amount of influence a given agent has over another. In models that use nominal opinion schemes, social influence cannot be implemented as averaging (Flache et al. 2017). These sorts of models tend to either rely on imitation dynamics, whereby an agent adopts one of the opinions of one of their contacts, or they rely on "majority rule" type dynamics, whereby an agent adopts the most frequent (sometimes weighted) opinion of her neighbors (Flache et al. 2017).

The second class of models identified by Flache et al. (2017) are models of similarity biased influence. In these sorts of models, agents will only work to reduce opinion differences with agents of whom they are 
"sufficiently similar" (Flache et al. 2017). The metric or criterion of similarity used to determine the scale of social influence depends on the underlying social or psychological theory underpinning the model. If the bias effect is sufficiently strong, we tend to observe the macro outcome of stable opinion clusters between agents (Flache et al. 2017). The basic assumption underlying these sorts of models is that agents will not be able to influence each other if their level of 'disagreement' is too high (Flache et al. 2017). Flache et al. (2017) identify two sorts of theoretical justifications for this class of models. The first sort of theoretical justification is one based on so-called 'bounded confidence'. These sorts of models tend to base themselves on cognitive theories of confirmation bias (Nickerson 1998) or social judgment theory (Flache et al. 2017; Maccoby 1962). In these sorts of models, the primary factor in whether or not an agent accepts an opinion or not depends on the attitude of the recipient toward the message (Flache et al. 2017). In other models, similarity is judged on the basis of the social relation between the influencer and the recipient. For example, Mark (1998) creates a model based on symbolic interactionism where individuals are more receptive to an influencer that is similar to them, regardless of the idea being propagated (Flache et al. 2017). Formal implementations of models using similarity bias with continuous opinions tend to look similar to models implementing assimilative influence with the caveat that opinion difference between the sender and receiver is only reduced in the case where the 'similarity criterion' is met. This can be similarly implemented in models with nominal opinions, such as Axelrod's (1997) famous model, where a receiving agent will only adopt opinions from agents with sufficient opinion overlap, ie: the number of traits/opinions in common.

The third class of models identified by Flache et al. are models of repulsive influence (2017). These sorts of models are similar to models of similarity biased influence, with the addition that not only are agents not influenced by those agents who are sufficently dissimiliar to them, but they actively become more dissimilar to agents that are different than them (hense the repulsive aspect). When disagreement between a given pair of agents reaches a critical level, the influence switches from a "pull" (difference reduction) to a "push" (dissimilarity increase) (Flache et al. 2017). The typical macro behaviour observed in this class of models is one of opinion bi-polarization. Stable opinion clusters are eventually disrupted by extreme agents pushing the moderate opinions held in the opinion clusters to one of the polls (Flache et al. 2017). Models of repulsive influence also use theories like cognitive dissonance reduction (Festinger 1957) to justify their models, however in this case dissonance reduction is interpreted not only to mean becoming more similar to those ideas that you like, but becoming more dissimilar to opinions that you don't like (Flache et al. 2017). Ideas such as xenophobia (E. Rosenbaum 1986), where individuals tend to react negatively to those that are dissimilar to them also motivate models of repulsive influence (Flache et al. 2017).

There are many models of social influence that fall outside of these discrete categories, however these classes give a broad overview of the major kinds of models one tends to find in the literature.

\section{The Internet: Challenges and Opportunities}

The models mentioned in the previous section are able to provide broad coverage over the major macro outcomes of social influence that we observe in everyday life: Consensus, opinion clustering, and bi-polarization. The theoretical justifications used to underpin such models rely on more traditional psychological and sociological theories such as cognitive dissonance reduction, confirmation bias, symbolic interactionism, and conformity effects. While these justifications are reasonable in a face to face setting, some considerations will have to be made for modelers wishing to study social influence online. On the internet, a new kind of underlying dynamics is at play that can at times complement, and at other times lie contradictory to, the dynamics of models geared for more traditional settings. On the other token, the internet provides many potential opportunities for modelers wishing to study social influence. Here we enumerate some "talking points" where challenges and opportunities for researchers are discussed for each point with the hope of inspiring directions for future research.

\section{One-to-one Communication vs. One-to-many Communication}


An important dynamic that emerges in online social contexts that tends to be more rare (and thus ignored by modelers of social influence) in traditional 'face to face' contexts is the idea of information spreading in a one-to-many fashion. Online, outside of private chat services, information is rarely communicated to a single recipient. Instead, information on the internet tends to flow to several recipients at once tweets are propagated to all followers of the tweeter, facebook statuses are made visible to all friends of the sharer, and youtube videos made visible for all subscribers of the content creator, etc. Keijzer et al. (2018) argue that this mode of communication can significantly alter the information dynamics of the system. Converting Axelrod's (1997) traditional model of cultural diffusion which uses a one-toone communication scheme to a one-to-many communication scheme, Keijzer et al. (2018) showed that such a scheme could increase both cultural isolation and opinion clustering among agents. As their work shows, it can be misleading to assume a traditional one-to-one communication structure in models of online settings. Researchers should take care to pick the appropriate communication structure that best matches their environment of study.

\section{Opportunities for Future Research}

The theoretical analysis done by Keijzer et al. (2018) would be well supported by more empirical research which studies the effects of one-to-many communication in real online environments. Models using different communication paradigms could be compared for their ability to cover empirical data, giving credence to the importance of communication structure in social influence.

\section{Cyberpsychology vs. Traditional Psychology}

A growing body of literature suggests that people tend to act differently in online social contexts than they do in face to face interaction. A relatively recent sub-field of psycholgy, known as cyberpsychology, aims to study these differences. Effects such as the 'Online Disinhibition Effect' (Suler 2004), where individuals tend to display a lowering of behavioural inhibitions in online environments, have been used to explain some of the unique behaviour observed in humans online. Likewise, phenomena such as trolls (users posting to incite emotional reactions) (Buckels, Trapnell and Paulhus 2014) or 'lurkers' (individuals that only read but do not propogate content) (Sun, Rau and Ma 2014) simply do not exist in offline social contexts. There is some research that suggests that computer mediated discussion can reduce inter-group prejudice and foster consensus formation (Schumann et al. 2017) compared to face-to-face settings. Traditional psychological models used to motivate models of social influence and polarization may not have the same ecological validity in online environments. Researchers wishing to study models of social influence in online contexts should be sure to use psychological justifications that are valid for their environment of study.

\section{Opportunities for Future Research}

More empirical and theoretical work is needed to understand how the underlying 'cyberpsychology' present in online settings can change the dynamics of social influence and lead to different models of polarization. Effects such as the online dishinbition effect could be incorporated into contemporary models of social influence by modulating the amount of information individual users share amongst each other. Likewise lurkers could be incorporated into models of social influence by introducing agents who can receive information but rarely propagate it. The implementation of so-called trolls into possible models of social influence is discussed in section 5.

\section{Static vs. Dynamic Social Networks}

Traditional models of social influence tend to focus on static social networks, where all network connections are set at the beginning of the simulation and left static until it's completion. Research suggests that social ties in online communities evolve over time (Backstrom et al. 2006). Likewise, the strength of social ties between users also changes dynamically (Viswanath et al. 2009). Models that take some of these dynamic network properties into account find that they can be crucial in stabilizing network polarization despite factors which tend to increase network convergence (Centola et al. 2007). Going forward, it will be important for modelers to identify which properties of dynamic networks are important in help- 
ing foster network polarization (A. Keijzer, Mäs and Flache 2018) and for researchers to empirically determine the kind of network evolution that actually occurs online .

\section{Opportunities for Future Research}

Recent literature on the problem of link prediction in social networks can help inform modelers of social influence how to integrate dynamic social networks into their models. If empirical analysis suggests that a particular link prediction model is well suited for a given social network domain, then social influence modelers can use this to motivate implementations of dynamic networks in their own domain.

\section{Recommender Systems and Algorthimic Curation}

Deemed the era of personalization, it has been known for some time that search engines and social networking websites will filter and curate the information seen by their users (Pariser 2011). Counter intuitively, some research suggests that algorithmic recommender systems may work to diversify user interests and increase the diversity of shared content in certain domains (Hosanagar et al. 2014; Nguyen et al. 2014). Recommender systems not only play a role in determining which content to show the user, but also may effect the social ties formed by users, for example in the recommendation of friends on Facebook (Lü et al. 2012). These sorts of algorithms pose a challenge for social influence researchers since now underlying website algorithms could play a significant role in shaping the opinion dynamics of a network. Empirical work is needed to determine how different classes of recommender systems and under what conditions they might help foster opinion consensus or polarization.

\section{Opportunities for Future Research}

Some researchers have begun to study the effect of algorithmic recommender systems in agent-based models. In particular, Geschke et al (2019) integrated two sorts of recommender systems into an ABM to study their effects. By artificially exposing curated content to the agents, these algorithmic recommender systems could be simulated to see their effect on macro outcomes. Although their model is a step in the right direction, the recommender system implemented in the model is rather rudimentary, making it hard to assess it's ecological validity. Implementing more realistic recommender systems into ABMs to study their effects could help shed light on the role these algorithms play in the domain of social influence.

\section{Trolls and Bots}

Recent events involving foreign influence campaigns online have sparked public interest in understanding the role of bots and trolls in shaping popular opinion (Zannettou et al. 2018). Bots and trolls differ in the sense that bots are online users which are controlled by automated systems, while trolls tend to be human controlled users. They are similar in the fact that their primary objective is in artificially spreading some target information. Recent empirical literature suggests that a minority opinion can usurp a stable majority opinion if the 'committed group' (the agents or users that do not change their opinion and only try to propagate their own) make up as little as $25 \%$ of the total population (Centola et al. 2018). This suggests that in the case that bots and trolls are indistinguishable from average users online, they could play a powerful role in shaping opinion dynamics.

\section{Opportunities for Future Research}

Further empirical and theoretical work is needed to determine the possible role these sorts of users can have in shaping public opinion. Through the use of publicly available datasets (Mehta 2018), researchers can study the effects and behaviours of real trolls online to construct more realistic models. Social network analysis of these datasets can also give some empirical verification to the findings of researchers like Centola et. al (2018).

\section{Conclusion}

In the first part of the paper, we provided historical context for the study of social influence by tracing back it's roots to early diffusion theorists. We then gave a broad overview of modern ABMs used to explain social 
influence by exploring 3 classes of models: models of assimilative social influence, models with similarity bias, and models of repulsive social influence. We concluded with a discussion of what ingredients these models are missing to fully accommodate them for the "internet age" and discussed ways in which modelers could add additional ingredients to better align them for this domain.

This is an extremely broad and vibrant area of research with plenty of opportunity for researchers to dig deep into any of the above listed topics. While the internet can no longer be considered "new", it's role in the domain of social influence is only just starting to be understood. Likewise, the amount of data now available online makes it easier than ever for modelers to empirically validate their findings or model assumptions. As we've discussed, the internet introduces new elements into the dynamics of social influence. It is our hope that researchers will take these points into consideration for their future undertakings in the field.

\section{References}

A. Keijzer, Marijn, Michael Mäs and Andreas Flache. 2018. 'Communication in Online Social Networks Fosters Cultural Isolation'. Complexity 2018 (): 1-18. doi:10 .1155/2018/9502872.

Axelrod, Robert. 1997. 'The Dissemination of Culture: A Model with Local Convergence and Global Polarization'. Journal of Conflict Resolution 41 (2): 203-226. doi:10 .1177/0022002797041002001. eprint: https: / / doi.org/10.1177/0022002797041002001.https://doi.org/10.1177/ 0022002797041002001.

Backstrom, Lars, et al. 2006. 'Group Formation in Large Social Networks: Membership, Growth, and Evolution'. In Proceedings of the 12th ACM SIGKDD International Conference on Knowledge Discovery and Data Mining, 44-54. KDD '06. Philadelphia, PA, USA: ACM. ISBN: 1-59593-339-5. doi:10 . 1145 / 1150402.1150412.http://doi.acm.org/10.1145/1150402.1150412.

Bonabeau, Eric. 2002. 'Agent-based modeling: Methods and techniques for simulating human systems'. Proceedings of the National Academy of Sciences 99 (suppl 3): 7280-7287. ISSN: 0027-8424. doi:10 . 1073 / pnas.082080899. eprint: https: / /www.pnas.org/content/99/suppl_3/7280. full. pdf. https://www.pnas.org/content/99/suppl_3/7280.

Buckels, Erin E., Paul D. Trapnell and Delroy L. Paulhus. 2014. 'Trolls just want to have fun'. The Dark Triad of Personality, Personality and Individual Differences 67:97-102. ISSN: 0191-8869. doi:https : // doi.org/10.1016/j.paid.2014.01.016. http:// www. sciencedirect. com / science/article/pii/s0191886914000324.

Centola, Damon, et al. 2007. 'Homophily, Cultural Drift, and the Co-Evolution of Cultural Groups'. Journal of Conflict Resolution 51 (6): 905-929. doi:10 .1177/0022002707307632. eprint: https : / doi . org/10.1177/0022002707307632. https://doi.org/10.1177/0022002707307632.

Centola, Damon, et al. 2018. 'Experimental evidence for tipping points in social convention'. Science 360 (): 1116-1119. doi:10.1126/science.aas8827.

E. Rosenbaum, Milton. 1986. 'The Repulsion Hypothesis. On the Nondevelopment of Relationships'. Journal of Personality and Social Psychology 51 (): 1156-1166. doi:10 .1037/0022-3514.51.6.1156.

Festinger, Leon. 1957. A Theory of Cognitive Dissonance. Stanford University Press. ISBN: 0804709114. http: //www. amazon.com/exec/obidos/redirect?tag=citeulike07-20\%5C\&path=ASIN/ 0804709114.

Flache, Andreas, et al. 2017. 'Models of Social Influence: Towards the Next Frontiers'. Journal of Artificial Societies and Social Simulation 20 (4): 2. ISSN: 1460-7425. doi:10 . 18564 / jasss . 3521. http : //jasss.soc.surrey.ac.uk/20/4/2.html. 
Geschke, Daniel, Jan Lorenz and Peter Holtz. 2019. 'The triple-filter bubble: Using agent-based modelling to test a meta-theoretical framework for the emergence of filter bubbles and echo chambers'. British Journal of Social Psychology 58 (1): 129-149. doi:10.1111/bjso.12286. eprint: https: / / onl inelibrary. wiley.com/doi/pdf/10.1111/bjso.12286. https://onlinelibrary.wiley.com/ doi/abs/10.1111/bjso.12286.

Hosanagar, Kartik, et al. 2014. 'Will the Global Village Fracture Into Tribes? Recommender Systems and Their Effects on Consumer Fragmentation'. Management Science 60 (): 805-823. doi:10.1287/mnsc . 2013. 1808.

Kinnunen, Jussi. 1996. 'Gabriel Tarde as a Founding Father of Innovation Diffusion Research'. Acta Sociologica 39 (4): 431-442. doi:10 .1177 / 000169939603900404 . eprint: https : / / doi .org/10 .1177/ 000169939603900404. https://doi.org/10.1177/000169939603900404.

Lü, Linyuan, et al. 2012. 'Recommender systems'. Recommender Systems, Physics Reports 519 (1): 1-49. ISSN: 0370-1573. doi:https : / / doi .org/10.1016/j . physrep.2012.02.006. http : //www.sciencedirect.com/science/article/pii/s0370157312000828.

Maccoby, Nathan. 1962. 'Social Judgment: Assimilation and Contrast Effects in Communication and Attitude Change. Muzafer Sherif, Carl I. Hovland'. American Journal of Sociology 68 (1): 126-127. doi:10. $1086 /$ 223278. eprint: https : / / doi .org/10.1086/223278. https : / / doi .org/10.1086/ 223278.

Mark, Noah. 1998. 'Beyond Individual Differences: Social Differentiation from First Principles'. American Sociological Review 63 (3): 309-330. ISSN: 00031224. http: / / www. jstor.org/stable/2657552.

Mehta, Dhrumil. 2018. Russian Troll Tweets. https://github.com/fivethirtyeight/russiantroll-tweets.

Nguyen, Tien T., et al. 2014. 'Exploring the Filter Bubble: The Effect of Using Recommender Systems on Content Diversity'. In Proceedings of the 23rd International Conference on World Wide Web, 677-686. WWW'14. Seoul, Korea: ACM. ISBN: 978-1-4503-2744-2. doi:10.1145/2566486.2568012. http: //doi.acm.org/10.1145/2566486.2568012.

Nickerson, Raymond. 1998. 'Confirmation Bias: A Ubiquitous Phenomenon in Many Guises'. Review of General Psychology 2 (): 175-220. doi:10.1037/1089-2680.2.2.175.

Pariser, Eli. 2011. The Filter Bubble: What the Internet Is Hiding from You. Penguin Group, The. ISBN: 1594203008, 9781594203008.

Rogers, Everett. 1963. 'Diffusion of innovations.The Free Press of Glencoe Division of The Macmillan Co., 60 Fifth Avenue, New York 11, N. Y., 1962.' Journal of Pharmaceutical Sciences 52 (6): 612-612. doi:10 . 1002 / jps. 2600520633. eprint: https: / / onlinelibrary .wiley . com/doi / pdf / 10 . 1002 / jps.2600520633. https:// onlinelibrary.wiley.com/doi/abs / 10 . 1002 / jps. 2600520633 .

Ryan, B., and N. Gross. 1943. 'The diffusion of hybrid seed corn in two Iowa communities'. Rural Sociology 8 (1): 15-24.

Schumann, Sandy, et al. 2017. 'When is computer-mediated intergroup contact most promising? Examining the effect of out-group members' anonymity on prejudice'. Computers in Human Behavior 77:198-210. ISSN: 0747-5632. doi:https : / / doi .org/10.1016/j.chb.2017.08.006. http: //www . sciencedirect.com/science/article/pii/s0747563217304739.

Suler, John. 2004. 'The Online Disinhibition Effect'. PMID: 15257832, CyberPsychology \& Behavior 7 (3): 321-326. doi:10.1089/1094931041291295. eprint: https://doi.org/10.1089/1094931041291295. https://doi.org/10.1089/1094931041291295.

Sun, Na, Patrick Pei-Luen Rau and Liang Ma. 2014. 'Understanding lurkers in online communities: A literature review'. Computers in Human Behavior 38:110-117. ISSN: 0747-5632. doi:https : / / doi . org / 10 . 1016/j.chb.2014.05.022. http://www.sciencedirect.com/science/article/ pii/s0747563214003008. 
Thomas W. Valente, Everett M. Rogers. 1995. 'The Origins and Development of the Diffusion of Innovations Paradigm as an Example of Scientific Growth'. PMID: 12319357, Science Communication 16 (3): 242-273. doi:10 . 1177 / 1075547095016003002. eprint: https : / / doi . org / 10 . 1177/ 1075547095016003002 . https://doi.org/10.1177/1075547095016003002.

Viswanath, Bimal, et al. 2009. 'On the Evolution of User Interaction in Facebook'. In Proceedings of the 2Nd ACM Workshop on Online Social Networks, 37-42. WOSN '09. Barcelona, Spain: ACM. ISBN: 978-160558-445-4. doi:10 .1145/1592665.1592675. http: / / doi . acm.org/10.1145/1592665. 1592675.

Zannettou, Savvas, et al. 2018. 'Disinformation Warfare: Understanding State-Sponsored Trolls on Twitter and Their Influence on the Web'. CoRR abs/1801.09288. 